\title{
Spatial Distribution and Clusters of Logistics Industry Nearby HSR Station-The case of Fuzhou Station
}

\author{
Xiulian Hu*, Qinding Zheng, Chong Ye \\ School of Economics and Management, Fuzhou University of International Studies and Trade, \\ Yuhuan Road, Fuzhou, China \\ Huxiulian2007@163.com
}

Keywords: HSRway, Logistics industry, Industrial cluster, Spatial distribution.

\begin{abstract}
Taking Fuzhou Station as an example, this paper regards logistics enterprises within the scale of research as points within this space and establishes point maps. Then it uses spatial analysis methods and Ripley's $\mathrm{K}$ function analysis and kernel density estimation to Analyze the spatial distribution and agglomeration characteristics of logistics industry in Fuzhou HSRway station. This paper focuses on the spatial distribution and agglomeration characteristics of the logistics industry in the high-speed railway station area, which has certain practical significance for the production service industry planning of the high-speed railway station area.
\end{abstract}

\section{Introduction}

HSR(HSR)allows people, finances, materials, and information to flow quickly. When a city has newly built a HSR station, its nearby areas will attract industries such as real estate and commerce. Just like the Japanese Shinkansen has brought several cities together and formed an industrial zone (Ning Guangjing, 1998). There have been a lot of studies on HSR or industrial agglomerations, but they are more concerned about their respective influences, such as the impact of HSR on urban spatial structure and the spillover effect of industrial agglomeration, and few people have studied the agglomeration effect of HSR on the logistics industry. Wang Li et al. used spatial analysis methods to explore the distribution and spatial agglomeration characteristics of the high-speed railway station industry. $\mathrm{L}$ Chen, XL Li believes that high-speed rail can reduce space transaction costs, compression space and time distance and other positive effects. Chatman D G, Noland R B. Transit Service pointed out that improvements in public transport may lead to more clusters and high-density employment, urban development, improved labor market access, increased information exchange and the promotion of industrial divisions leading to agglomeration economies.

The agglomeration of the logistics industry can promote the development of social economy. Analyze the spatial distribution and agglomeration characteristics of the logistics industry near the HSR station, the impact of the construction of the HSR on the surrounding industrial agglomeration and the influencing factors. Analysis of these can allow us to more scientifically plan for the logistics industry near the HSR. 


\section{Model introduction}

\subsection{Ripley's K Function}

Ripley's K method is a commonly used spatial point data analysis method. By using Ripley's K function, point data sets can be analyzed at different distances. This function is based on distance and indicates the degree of spatial aggregation or diffusion of points in a certain range. Ripley's $\mathrm{K}$ function is a cumulative distribution function, which has a cumulative effect on the large scale and complicates the pattern, but is widely applied on a small scale (Wang Li,2012). The HSR stations obviously belong to the latter, so this function applies. In this study, it can aggregate relevant information of point data of all logistics-related companies within a range of 3,000 meters for further agglomeration or decentralized analysis.

Definition: Set the enterprise point to $\mathrm{x}_{\mathrm{i}}$, where $\mathrm{N}_{\mathrm{d}}$ is the number of points $\mathrm{x}_{\mathrm{i}}$ contains in a circle with $\mathrm{d}$ as radius, and $\mathrm{d}$ is the distance, that is, the number of companies involved when the distance is less than or equal to $\mathrm{d}$. So given a different $\mathrm{d}$, the $\mathrm{K}$ function is:

$$
\mathrm{K}(\mathrm{d})=\frac{\pi}{2} \mathrm{E}\left(\mathrm{N}_{\mathrm{d}}\right)
$$

In the formula, $E\left(\mathrm{~N}_{\mathrm{d}}\right)$ represents the mathematical expectation of the company's neighbors in the circle with $\mathrm{x}_{\mathrm{i}}$ as the center and $\mathrm{d}$ as the radius. $\lambda$ represents the distribution density of the enterprise as a whole, which is a constant (Wang Li,2012). It is known that $n$ enterprises $x_{1}, x_{2}, x_{3}, \ldots X_{n}$ within a circle with a radius $d$ have an area of $\mathrm{R}$. To estimate the K-function value of the point-ofobservation distribution, the nearest neighbors of the company in the buffer zone with the distance $d$ can be calculated first, and then divided by the overall distribution density of the enterprise points in the study area (Wang Li,2012). The formula is as follows:

$$
\hat{K}(d)=\frac{1}{\lambda_{\mathbb{A}}} \sum_{i \neq \mathrm{j}} \sum_{n^{2}} I_{d}\left(h_{\mathrm{ij}}\right)
$$

In this formula, $\widetilde{K}(d)$ is the estimated value of $\mathrm{K}$ function, $\bar{\lambda}_{\bar{d}}^{-}$is the estimated value of $\lambda$, which can be approximately equal to $\mathrm{n}^{2} / \mathrm{R}$, and $h_{\mathrm{ii}}$ represents the linear distance between $\mathrm{x}_{1}$ and $\mathrm{x}_{2}$. Where $I_{d}\left(h_{\mathrm{ii}}\right)$ is defined by the following formula:

$$
I_{d}\left(h_{\mathrm{ij}}\right)= \begin{cases}1 & h_{\mathrm{ij}} \leq d \\ 0 & h_{\mathrm{ij}}<d\end{cases}
$$

When the point process obeys the complete spatial randomness of the homogeneous Poisson point process, the theoretical value of $\mathrm{K}(\mathrm{d})$ is equal to $\mathrm{md}^{2}$. Because of the limited ability of the graph of the K-function, refer to the L-function of Besag and do a linear transformation of $\mathrm{K}$ (d) (Besag J,1974) to maintain the stability of the variance.

$$
\mathrm{L}(\mathrm{d})=\sqrt{\frac{\mathrm{K}(\mathrm{d})}{\pi}}-\mathrm{d}
$$

Under the assumption of stochastic distribution, the expected value of $L(d)$ is 0 . When $L(d)>0$, it indicates that the enterprises are clustered and are attracted to each other. When $L(d)<0$, it means that enterprises are evenly distributed and mutually exclusive. When $\mathrm{L}(\mathrm{d})=0$, it means that companies are distributed independently.

In addition, the $\mathrm{L}$ function uses Monte Carlo simulation to test the significance. Simulating 100 times will produce a curve that represents the upper and lower limits of the confidence interval, ie, the maximum and minimum values of the $\mathrm{L}(\mathrm{d})$ simulation. With $\mathrm{d}$ as the abscissa, the upper and lower limits of the confidence interval are plotted as ordinates. Use real-world spatial data to derive different $\mathrm{L}(\mathrm{d})$ values. When $\mathrm{L}(\mathrm{d})$ is outside the confidence interval, it indicates that the relationship 
between the companies is significant.

\subsection{Kernel Density Estimation Method}

Kernel density analysis is to distribute the magnitude of each sampling point throughout the study area. Use the kernel function to calculate the value per unit area based on the point features to fit the point to a smooth conical surface, and then calculate the density value of each pixel in the output raster (Kangwen Zhu, 2016).

The kernel density estimation method studies the distribution characteristics of points by observing the spatial variation of point density in the range region (Rui Liu,2011). The data in the enterprise point map is discrete, and the kernel density estimation can convert the originally discrete data into continuous variables. Therefore, it is appropriate to use kernel density estimation in this paper. The kernel function can be used to estimate the theoretical spatial density of logistics companies in Fuzhou station area. Then according to the density can draw a contour map. The formula is as follows.

$$
\widehat{\lambda_{d}}(\chi)=\frac{3}{\pi d^{2}}\left[1-\left(\frac{a_{1 j}}{d^{2}}\right)^{2}\right]^{2}
$$

Where $\bar{\lambda}_{d}^{-}(x)$ is an estimate of $\lambda_{d}(x)$ and $\mathrm{K}(\cdot)$ is a kernel function. $\lambda_{d}(x)$ calculates the density of $\mathrm{x}$ by calculating the number of firm points per unit area within the target range. $X=\{x 1, x 2, x 3, \ldots x n\}$ indicates a set of business points. $\mathrm{Xi}$ is the position of the $\mathrm{i}$-th enterprise within a circle with $\mathrm{x}$ as its center and d as its radius. $d$ represents the bandwidth, which has an effect on the smoothness of the density estimate. With the increase of $d$, the density of points on the space changes more smoothly, but the structure of the density will be covered; as the value of d decreases, the estimated density of points changes abruptly (Tao Wang,2012).

In this study, the fourth-order polynomial in kernel density estimation is used to calculate, the formula as follows:

$$
\widehat{\lambda_{d}}(\chi)=\frac{3}{\pi d^{2}}\left[1-\left(\frac{a_{11}}{d^{2}}\right)^{2}\right]^{2}
$$

Where $a_{i j}$ represents the euclidean distance from point $\mathrm{i}$ to point $\mathrm{j}$.

\section{General Features Overview and Assumptions}

Near the HSR station area, the spatial layout of the logistics industry is mostly spread out with the HSR station as the center, and the specific location of the layout is closely related to the logistics channel network. The logistics channels here are mostly road networks and subway networks. The aggregation of logistics industry has its own choice and consideration criteria for geographical location. The transport industry in the logistics industry is mostly based on or close to roads, especially main roads or transportation hubs. The storage company is more concerned about land prices, because such companies need a large area of land space, higher land requirements. Storage company, especially those responsible for the last mile, are mostly close to residential areas.

Of course, the factors influencing the layout of the logistics industry in the HSR station area include: the level of economic development, the infrastructure surrounding the HSR station, topography and terrain, and urban land use. The influence of these factors on the distribution of logistics industry is very significant, and sometimes even destroys the original layout rules of the industry.

In the HSR station area, the distribution of logistics industry usually has a significant 
agglomeration feature. While the HSR station attracts the logistics industry to gather here, these clustered logistics industries also improve the conditions surrounding the HSR station, which in turn attracts other industries such as manufacturing and distribution industries. The convergence of the logistics industry has the characteristics of dependence. It provides services for social activities to meet the needs of society. Cultivating and supporting other industries will bring about the growth of the animal flow industry, especially in industries such as manufacturing that have huge logistics needs. When the logistics demand generated by these industries continues to grow and gather, the development of the logistics industry will also increase.

In terms of the distribution characteristics of the logistics industry near the HSR station, this paper makes two assumptions. First, the logistics industry near the HSR station is characterized by a circular structure. With HSR stations as the center, related companies expand outward and converge. Second, the gathering of logistics industry near the HSR station will develop towards the logistics park. Assume that when the logistics companies attract each other, they will have a scale effect within a certain area and then establish a logistics park. This is the best way to support the convergence of the logistics industry. At the same time, logistics parks usually have some preferential policies to support the development of logistics companies. Of course, they can also make greater use of logistics infrastructure.

Table 1 Assumptions of distribution

\begin{tabular}{|c|c|}
\hline Assumption & specific description \\
\hline $\mathrm{H}_{1}$ & $\begin{array}{c}\text { The logistics industry near the HSR station is } \\
\text { characterized by a circular structure. }\end{array}$ \\
\hline $\mathrm{H}_{2}$ & $\begin{array}{c}\text { The clustering of the logistics industry near the } \\
\text { HSR station will develop toward the logistics park. }\end{array}$ \\
\hline
\end{tabular}

\section{Analysis on Spatial Distribution and Agglomeration Characteristics of Logistics Industry in HSR Station Area of Fuzhou City}

\subsection{Data collection and Spatial Processing.}

The scope of the HSR station area is centered on the HSR station and extends outward into the loop structure. The data studied in this paper comes from the electronic map of Fuzhou City on the Baidu map, from which we can find logistics-related companies. In ArcGIS 10.2, the spatial information of the map of Fuzhou City was used to manually match each enterprise and establish a map of the enterprise space space related to the logistics industry, as shown in Figure 1. The database includes 99 enterprises that expand outwards within 3000 meters from the center of Fuzhou station, and also provides information such as company name and address.

\subsection{Spatial Distribution Characteristics of Logistics Industry}

In defining the scope of influence of the HSR station, the area with the station as the core and a 10-minute "comfortable walking distance" of 500 meters as the radius is usually used as the boundary (Guozhi Lin,2015). Therefore, in order to discuss the distribution characteristics of the logistics industry in the HSRway station area, Fuzhou Station is taken as an example to first analyze the density distribution of the circle, as shown in Figure 2. The circle is selected as 500 meters, 1000 meters, 1500 meters, 2000 meters, 2500 meters and 3000 meters as the buffer radius. 


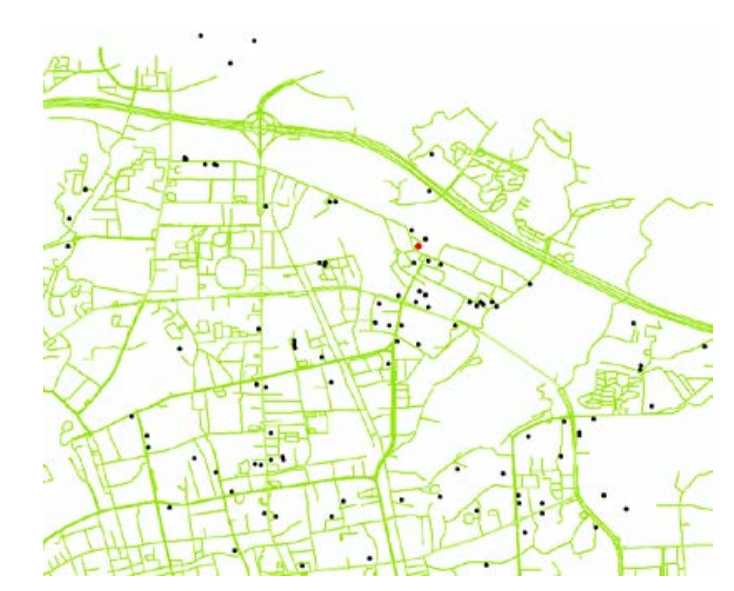

Figure 1 Distribution of logistics enterprises in Fuzhou Station

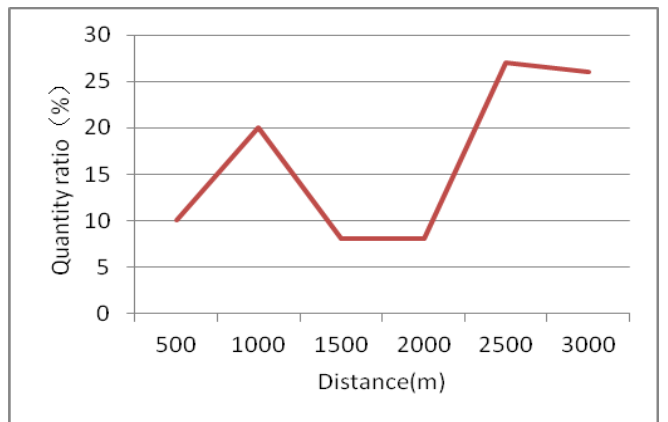

Figure 2 Distribution of the density of the circle in the logistics industry in Fuzhou station area

In general, from the perspective of layout, the logistics industry near the HSR station has a significant circle structure. The higher degree of agglomeration is concentrated at the circle of 2500 meters and 3000 meters.

In the 500 meters circle, due to the proximity to HSR stations, the logistics companies here are mostly transport companies, such as passenger terminals and automobile transportation, in order to connect with HSR transport and facilitate the conversion of different modes of transport. In the 1000-meter circle, because of relatively close distance from the HSR station, and the rent is also cheaper than the 500-meter circle, the company established there is also relatively more comprehensive.In addition, due to the influence of the construction of the Fuzhou Metro, there are also many companies that set up subways in this circle. As the distance increases, the area of residential housing also increases. In the circle of 1500 meters and the circle of 2000 meters, the area of the community accounts for most of the area, so the logistics enterprises in these two places also decreased. Of course, most of the companies set up in these circles are express delivery companies. They use more end-to-end delivery to solve the last kilometer problem so as to provide better services. In the circle of 2500 meters and the circle of 3000 meters, the number of enterprises is the most distributed.In the south of Third Ring Road, the circle extending from the center of the HSR station to the west, the south, and the east is closer to the center of the city, which in turn makes the two forms of activities within the circle more frequent and closer to the business center. The economic, cultural, and other aspects are also more prosperous. So service logistics companies have also increased. In the north of the Third Ring Road, most of them are industrial parks. Therefore, there are many warehousing companies with large demand for land.

The assumptions in the overview of the previous general characteristics can be confirmed by the analysis of the structure of the circle. Logistics companies expand and converge around HSR stations. The logistics industry near Fuzhou Station has shown a remarkable circular structure. 
Moreover, due to their own specific reasons, such as the level of economic development and urban land use, each circle has some differences in the spatial distribution characteristics. This is exactly the same factor as the impact of the layout of the logistics industry in the HSR station area mentioned in the previous overview.

\subsection{The spatial Agglomeration Characteristics of Logistics Industry}

Through the Ripley's K function in the special space point pattern analysis software Crimestat3.3, the established enterprise space point data related to the logistics industry is analyzed. The results are shown in Figure 3.

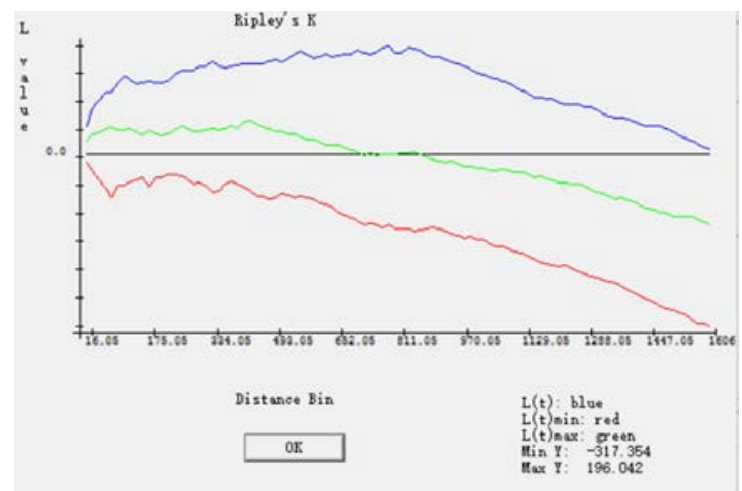

Figure 3 Ripley's K Function in Logistics Industry in Fuzhou Station Area

The conclusion shows that these data have passed the significance test. And the concentration of logistics industry near Fuzhou Station is greater than the maximum value of random distribution, that is, higher than the upper limit of confidence interval. Explain that these spatial point data are significantly agglomerated within the specified range. Furthermore, it also proved that the spatial agglomeration of logistics companies in the HSR station area is widespread. From Figure 3, we can see that Ripley's L is positive, lies outside the upper and lower confidence intervals L_max and L_min, and is always above the L_max curve, indicating that the logistics industry is significantly clustered near the HSR station area. The L curve first rises and then falls, and the distance between L and L_max also increases first and then decreases, reaching a maximum around 811.05 meters. This shows that between 0 and 811.05 meters, as the distance increases, the degree of agglomeration also increases, and then as the distance increases, the degree of agglomeration decreases.

After analysis, the distribution characteristics of the logistics industry near Fuzhou Station are consistent with the previously mentioned logistics industry distribution that has a significant agglomeration characteristic.

\subsection{Spatial Distribution Characteristics of Logistics Industry}

The use of space-based maps of logistics industry-related companies for nuclear density analysis will further analyze the spatial distribution characteristics of the logistics industry. Using the kernel density estimation and analysis method in ArcGIS 10.2 software to compare the spatial aggregation and distribution of the logistics industry, the distribution characteristics of the logistics industry in Fuzhou Station Area are plotted, as shown in Figure 4. 


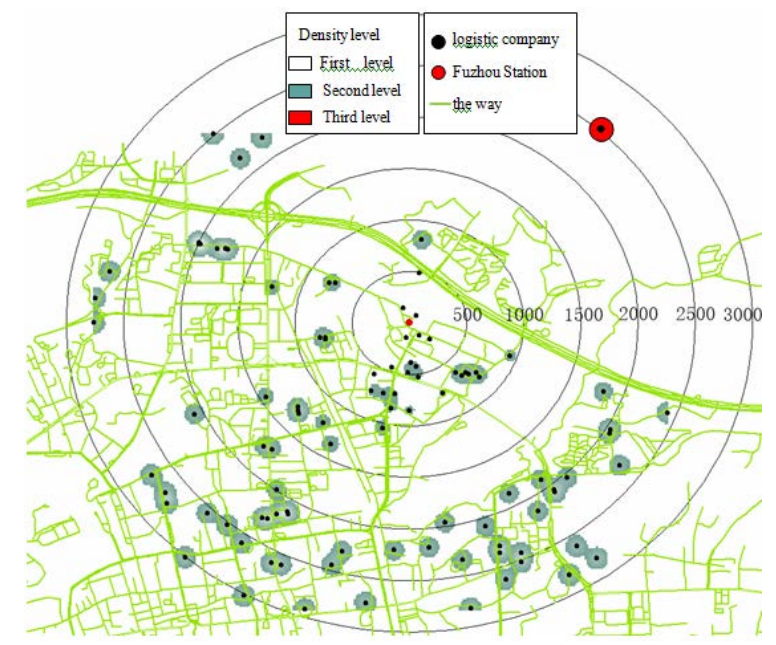

Figure 4 Estimation of nuclear density of logistics industry in Fuzhou Station

The figure shows that the degree of agglomeration is consistent with the focus of economic development. The closer to the core area of the city, the higher the kernel density. The agglomeration characteristics of the logistics industry show that there are many agglomeration hotspots and the scope involved is relatively large. The spatial point layout is also relatively balanced.

In general, the surrounding areas of Fuzhou Station were affected by the topography of Gushan, Jinjishan, Dengyun Reservoir, and Qinting Lake, which caused the distribution of logistics companies to be fragmented when they encountered this type of terrain. Among them, Dengyun Reservoir and Gushan were the most obvious. Dengyun Reservoir and Gushan are located in the northwest of Fuzhou Station, which makes the logistics enterprises in the northwest of Fuzhou Station almost empty. Fuzhou Station is located about 500 meters south of the fast loop of Sanhuan. Therefore, the northern and northwestern areas of the Third Ring Road are far from the city center, and there are relatively few logistics companies here. In the southeastern part of it, due to the presence of Jinjishan in the 1500-meter circle, there are faults in the circle enterprises, but there are more enterprises in the 1000 meters, 2000 meters, and 2500 meters circle layers, of which the 2500 meters circle layer has the most significant degree of enterprise agglomeration. Most of the layout along Lianjiang North Road and Changle North Road. Fuzhou Station is surrounded by the Metro Line 1 and Dongpu Tributary Road, Hualin Road, Zhanqian Road, North Ring Road, and other road networks. The south is close to the city center and there are more companies. It is worth mentioning that most of the 1000 meters circle is located along the 61 North Road. The enterprises in each circle in the South West have layouts, of which the concentration of enterprises is most obvious at the circle of 2500 meters and 3000 meters. The South West is connected with the Gulou District, which is also a high-aggregation area.

The distribution of the logistics industry near Fuzhou Station is affected by the topography and terrain, which is exactly the same as the impact factors in the logistics industry layout of the HSR station mentioned above. Furthermore, the distribution of logistics industry near Fuzhou Station just verified that the spatial distribution of logistics industry mentioned above was mostly spread out with HSR stations as the center, and the specific location of the layout was closely related to the logistics channel network.

However, there are no logistics parks in the Fuzhou station area, and its development has not developed towards the logistics park. This differs from the previous assumptions. The main reason for this is that the construction of Fuzhou station was earlier, and the surrounding land was also relatively tight, and it was unable to meet the demand for a logistics park with a large area. In 
addition, affected by the topography, terrain, highway network, and other industries near Fuzhou Station, it failed to meet the conditions required for the formation of a logistics park.

\section{Suggestions on the Development of Logistics Industry in HSR Station Area}

First, the agglomeration of logistics industry can promote the sustained growth of regional economy, reduce the operating costs of enterprises, expand the market scale, and enhance competitiveness. The logistics industry is clustered in the high-speed rail station area. When planning for the logistics industry, each province and city should fully consider the impact of the high-speed rail station area on the logistics industry and reasonably determine its development goals so that the two can promote each other. Realize the coordinated development of high-speed railway stations and logistics industry. And we must face up to the current system of logistics management issues such as long management, management, and other issues, the government should play a regulatory role as a whole.

Second, the government should not interfere excessively with the development of the logistics industry. In particular, the regulation of railway transportation can be gradually relaxed. At the same time, attention should be paid to the integration of transportation and logistics, which is more conducive to the gathering of the logistics industry in the high-speed rail station area. In recent years, although China's logistics industry has developed rapidly, there are problems with the uncoordinated development and the division of departments and regions. Therefore, when planning the various industries in the high-speed railway station area, each province and city can appropriately strengthen the spatial structure of the logistics industry, rationally improve the distribution of the logistics industry, and improve the interaction between the two so that they can play a specialized role in gathering and promote the development of the national economy.

Third, under the guidance of the government, integrate existing logistics resources and strengthen the construction of infrastructure near high-speed railway stations to promote the effective use of resources and promote the development of the logistics industry. Through the establishment of public logistics information platform to promote resource sharing, improve the efficiency of logistics operations. Of course, it can also promote the construction of enterprise informatization and promote the communication between enterprises. These integration measures can increase the degree of agglomeration of the logistics industry.

Fourth, it cannot be ignored that the agglomeration of the logistics industry has also been affected by other industries, especially manufacturing. The degree of industrialization has affected the demand for logistics industry. Therefore, when planning industrial projects in the high-speed railway station area, we must take both measures into consideration and focus on achieving the linkage development between the two. The rational distribution of these two industries will not only make the logistics industry continue to grow, but the manufacturing industry will also be able to enjoy professional services, which of course reduces the overall logistics costs of the society.

Fifth, the modern logistics industry needs the support of information systems. Enterprises enhance their own competitiveness through information sharing, resource sharing, and technology exchange. The establishment and improvement of an information sharing system can promote the exchange and communication between the logistics industry in the high-speed railway station area, the logistics industry and other industries, and promote the development and agglomeration of the logistics industry in the high-speed railway station area. This is also one of the effective ways to develop logistics standardization.

\section{Conclusions}

This paper discusses the spatial distribution and agglomeration characteristics of the logistics 
industry in the high-speed railway station area of Fuzhou Station. The following conclusions are drawn: (1) With the Fuzhou station as the center, the area with a radius of 500 meters is used as the boundary, and six circles are delineated. In general, from the layout point of view, the logistics industry near the high-speed rail station has a significant circle structure. The company's density reached its highest level at 2,500 meters and 3,000 meters. (2) Logistics enterprises are significantly concentrated in the distances observed by the high-speed railway station area. (3) The accumulation of logistics industry in the high-speed railway station area has the characteristics of wide layout and balanced distribution.

\section{Acknowledgements}

This paper is supported by Fujian Provincial Department of Education Youth Project (JAS180725).

\section{References}

[1] Besag J, 1974. Spatial interaction and statistic alanalysis of lattice systems, J. Roy. Statist. Soc. B. (36) , pp. 192236.

[2] Guangjing Ning, 2009. China Speed in High-speed Railway Period, Railwav Purchase and Logistics. (12) , pp.2526.

[3] Guozhi Lin, 2015. Studay on development theory and planning practice of High-speed railway station areas -- A case study in Wenling, Zhejiang University of Technology.Zhejiang.

[4] Kangwen Zhu, 2016. Research on the Geographic Concentration Measurement and Spatial Temporal Evolution of the Manufacturing Industry in the Mountainous City Based on GIS, Chongqing Normal University. Chongqing.

[5] Rui Liu, Weiping Hu, Hongliang Wang, 2011. The Road Network Evolution of Guangzhou-Foshan Metropolitan Area Based on Kernel Density Estimation, Scientia Geographica Sinica. 31(1), pp. 81-86.

[6] Tao Wang, Yongjin Chen, Yunbo Ma, Yanxun Liu, 2012. Spatial Clustering Analysis of Tuberculosis in Linyi City of Shandong Province, Chinese Journal of Health Statistics. (05) , pp. 633-635.

[7] Wang Li,Youhui Cao, Kewen Liu, Cong Wang, 2012. Spatial Distribution and Clusters of Industry Nearby Highspeed Rail Station:The case of Nanjing Station, Shanghai-Nanjing Motor Train, Scientia Geographica Sinica. 32(3) , pp. 301-307. 